\title{
Elderly Patients Achieving Clinical and Radiological Outcomes Comparable with Those of Younger Patients Following Minimally Invasive Transforaminal Lumbar Interbody Fusion
}

\author{
Joshi Nikhil N., Joel Wei-An Lim, William Yeo, Wai-Mun Yue \\ Spine Service, Department of Orthopaedics, Singapore General Hospital, Singapore
}

\section{Study Design: Retrospective analysis of prospective database.}

Purpose: To compare 2-year clinical and radiological outcomes after minimally invasive transforaminal lumbar interbody fusion (MISTLIF) among "middle-age" (50-64.99 years), "young-old" (65-74.99 years), and "old-old" (>75 years) patients.

Overview of Literature: Owing to higher perioperative morbidity and mortality rates, elderly patients with degenerative lumbar conditions are occasionally denied surgical care, even after conservative treatment failure. MIS-TLIF advantages include reduced blood loss, reduced analgesia requirements, early mobilization, and shorter hospital stays.

Methods: Between 2007 and 2012, 22 patients (age > 75 years) treated with 1-2 level MIS-TLIF were matched with "young-old" and "middle-age" patients (22 each) based on race, body mass index (BMI), diagnosis, spinal level, number of spinal levels operated upon, and bone graft type. Clinical outcomes included the Oswestry disability index (ODI), neurogenic symptom score (NSS), 36-item short form health survey (SF-36), and visual analogue scale (VAS) for back and leg pain. Radiological assessment included plain radiographs and preoperative magnetic resonance imaging (MRI) and plain radiographs at 1, 3, 6, and 24 months postoperatively. Fusion grade, loosening, cage migration, and adjacent segment degeneration were assessed.

Results: The groups had similar fluoroscopy time, operation duration, and postoperative analgesia type used. "Old-old" patients took longer to ambulate (1.6 days) and had longer hospital stays (6 days). All patients showed significant improvement in clinical outcome scores at all time-points compared with the preoperative status. "Middle-age" patients showed better ODI and SF-36 physical function scores than "old-old" patients preoperatively and 2 years post surgery. NSS, VAS (back and leg), and SF-36 mental function scores were similar between groups preoperatively and at every time-point postoperatively. Minimal clinical important differences $(63.6 \%-95.5 \%$ at 2 years) were achieved. Grade 1 fusion occurred in a minimum of $80 \%$ patients in each group 2 years post surgery. Complication rates were similar. Adjacent segment disease occurred in 2 patients from the "young-old" group, with no significant differences between groups. Conclusions: MIS-TLIF showed comparable results in selected "old-old" patients compared with "young-old" and "middle-age" patients without increased complication risks.

Keywords: Lumbar spine; Elderly patients; Minimally invasive transforaminal lumbar interbody fusion; Clinical outcomes; Minimal clinical important differences

Received Jun 28, 2016; Revised Aug 2, 2016; Accepted Aug 23, 2016

Corresponding author: Wai-Mun Yue

Department of Orthopaedic Surgery, Singapore General Hospital, Outram Road, 640633 Singapore

Tel: +65-63214603, Fax: +65-62248100, E-mail: yuewm@singnet.com.sg 


\section{Introduction}

As the geriatric population rises, the number of elderly patients presenting with painful lumbar degenerative pathology requiring fusion is expected to increase $[1,2]$. Traditional spine surgery involves neural decompression and fusion by using either posterolateral fusion (PLF) or interbody techniques via the posterior lumbar interbody fusion (PLIF) or transforaminal lumbar interbody fusion (TLIF) route. Current literature reports a high incidence of perioperative complications and increased morbidity and mortality in the elderly population when using these techniques [3-8]. Consequently, spinal surgeons are very hesitant in offering surgical care to elderly patients, even when conservative treatment fails to relieve their symptoms.

Minimally invasive surgery (MIS) TLIF has gained popularity in recent years due to the short and medium term clinical outcomes of this technique being comparable with those of open procedures. Moreover, MIS-TLIF has several advantages over open surgery, including reductions in blood loss, tissue trauma and analgesic requirement, and is associated with earlier mobilization from bed and shorter hospital stays [9-12].

We hypothesized that by offering MIS-TLIF surgery to appropriately selected elderly patients, we would be able to relieve them of debilitating pain and disability in a safe and effective manner. This study, therefore, aims to assess clinical and radiological outcomes in "young-old (65-74 years age)" and "old-old (>75 years age)" patients treated with MIS-TLIF two years previously and compare these with "middle-age (50-65 years age)" patients who had been similarly treated.

We hypothesized that there was no significant difference in terms of clinical outcome when "old-old" and "youngold" populations undergoing MIS-TLIF were compared with a similar patient population in the "middle-age" group.

To the best of our knowledge, very few studies have investigated the outcomes of MIS-TLIF in the elderly population, especially compared with other age groups $[13,14]$.

\section{Materials and Methods}

\section{Study design}

This study involved the retrospective analysis of a prospective database.

\section{Patient population}

Between 2007 and 2012, 363 patients were treated by the senior author (W.M.Y.) using MIS-TLIF in a tertiary institution. The study was carried out with the approval of the Institutional Review Board (IRB). Indications for surgery were persistent neurogenic symptoms with/without mechanical back pain which was refractory to conservative therapy for at least 6 months with correlated radiological features upon magnetic resonance imaging (MRI) and plain radiography. Inclusion criteria included all patients aged 50 years and older with preoperative diagnoses of degenerative spondylolisthesis (grade I/II) with/without degenerative scoliosis, lumbar spinal stenosis with instability, and degenerative disc disease with instability; all with a minimum 2-year follow up period. Exclusion criteria included patients with an age $<50$ years, and those with previous spinal surgery at the indexed level, spinal tumors, infection, fractures and patients with a follow up period $<2$ years. Our relative contraindications for MISTLIF included (1) high-grade (grade III/IV) spondylolisthesis; (2) severely collapsed disc space with no instability on dynamic radiographs; (3) patients who needed multilevel (more than 2 levels) decompression and fusion; (4) patients with combined coronal and/or sagittal deformities (kyphoscoliosis), and (5) patients who had back disease involving trauma, infection or other pathological causes.

Twenty-five patients aged $>75$ years, of which 3 patients were lost to follow up within first 2 years, were identified and formed the "old-old" group (22 patients). Two matching groups ("young-old" and "middle-age") were also identified based upon race, body mass index, preoperative diagnosis, spinal level of surgery, the number of surgery levels, the type of bone graft and the use of bone morphogenic protein (BMP). Thus, a total of 66 patients were included in our final analysis with 22 patients in each of the 3 study groups.

\section{Outcome measures}

Details of the MIS-TLIF operative technique were described in a previous publication from the same institute [9]. Demographic data for our patients were obtained by reviewing case sheets. Preoperative co-morbidities were assessed in terms of American Society of Anesthesiologists (ASA) grading obtained from anesthetic charts. 
Details of any epidural steroid injections (ESI) received by the patients were noted, along with the frequency of ESI and duration of the asymptomatic period following each injection. ESI included interlaminar epidural injection, targeted transforaminal ESI and caudal epidural ESI.

Postoperatively, early mobilization of patients was encouraged. Patients were considered fit for discharge to home only when they were able to ambulate independently for a minimum of 10 meters and could climb stairs, if necessary. We administered patient controlled analgesia or intramuscular pethidine for pain relief. For calculation, intramuscular pethidine was converted into morphine equivalents. Perioperative outcome measures included fluoroscopic time (seconds), operative time (minutes), morphine/analgesia used (mg), time to ambulation (days) and hospitalization required (days). Following discharge, patients were assessed by the primary surgeon at 2 weeks, 6 weeks and at 3 months, 6months, 12 months and 24 months.

Clinical outcome scores were assessed using the Oswestry disability index (ODI), North American Spine Society neurogenic symptom score (NSS), 36-item short form health survey (SF-36) and visual analogue scale scores (VAS) for back and leg pain. Minimal clinically important difference (MCID) values were calculated for all clinical outcome scores and the proportion (\%) of patients reaching MCID values were evaluated at every follow-up $[15,16]$. These scores were collected by two independent assessors not directly involved in patient care at the Orthopaedic Diagnostic Centre of our institution. This data were collected prospectively preoperatively and at each postoperative follow-up visit.

Postoperative complications following MIS-TLIF were recorded by reviewing case records. These were classified as "early" (within 28 days of surgery) and late (after 28 days of surgery) complications, and also classified as "major" and "minor" as per Lebude et al. [17]. A complication was considered major if it involved postoperative neurodeficit(s) or permanent loss of function. Medical complications which significantly prolonged hospital stay and revision surgery were also considered as major complications [17]. Minor complications included radiological abnormalities which may/may not be symptomatic and other conditions such as electrolyte imbalance, urinary tract infections (UTIs), superficial surgical site infections and dural tears which were successfully treated.

\section{Radiographic measurements}

Radiologic assessment included preoperative plain radiographs (static and dynamic erect views) and MRI scans of the lumbar spine. Radiographs of the lumbar spine were obtained at each follow-up visit to examine the extent of fusion and monitor for complications. Radiological assessment was performed by two independent observers who were not directly involved in patient care. Fusion rates were assessed according to the Bridwell classification [18]. Screw/Cage loosening was defined as radiolucency of $1 \mathrm{~mm}$ or more around the implant. Adjacent segment degeneration (ASD) was diagnosed when there was anterolisthesis/retrolisthesis $>4 \mathrm{~mm},>10^{\circ}$ of angular motion on dynamic films, $>10 \%$ loss of disc height, $>3 \mathrm{~mm}$ of osteophyte formation at the adjacent segments (up to 2 levels apart), degenerative scoliosis or compression fracture as described by Bae et al. [19] and Cheh et al. [20] on plain radiographs. ASD was divided into radiological and clinical ASD according to Kim et al. [13].

\section{Statistical analyses}

Statistical analyses were performed with SPSS ver. 21 (SPSS Inc.., Chicago, IL, USA). The Pearson chi-square test/Fisher exact test was used to compare categorical data (fusion grading, cage migration, ASD, and complication rates), while analysis of variance was used to compare continuous variables (length of operation, length of hospitalization stay, fluoroscopy time, amount of patientcontrolled analgesia and time to ambulation). Repeated measures was employed in the analysis of ODI, NSS, SF36 , and VAS back and leg pain. Significance was defined as $p<0.05$. Post hoc analyses were performed using Bonferroni's method. Cohen's kappa was also employed to determine inter-observer variation in radiological measurements.

\section{Results}

In total, there were 22 patients in each of the three study groups. Demographic profile and perioperative outcomes are described in Table 1. The most common diagnosis was degenerative spondylolisthesis (grade I/II) with stenosis while L4/5 was the most common level of surgery in all three groups. There were 14 cases of single level MIS-TLIF and 8 cases of double level surgery in each age 
Table 1. Demography and perioperative outcome measures

\begin{tabular}{|c|c|c|c|c|}
\hline Demographic criteria & $\begin{array}{l}\text { Old-old } \\
(n=22)\end{array}$ & $\begin{array}{l}\text { Young-old } \\
\qquad(n=22)\end{array}$ & $\begin{array}{l}\text { Middle-age } \\
\quad(n=22)\end{array}$ & $\begin{array}{c}\text { Level of } \\
\text { significance } \\
p \text {-value }\end{array}$ \\
\hline Age & $78.18 \pm 2.58^{*}$ & $69.99 \pm 2.56^{*}$ & $58.88 \pm 3.91^{*}$ & 0.005 \\
\hline Sex (male:female) & 18.2:81.8 & $22.7: 77.3$ & $22.7: 77.3$ & 0.913 \\
\hline Race & Chinese & Chinese & Chinese & NA \\
\hline $\mathrm{BMI}$ & $24.67 \pm 2.94$ & $24.57 \pm 2.61$ & $24.88 \pm 2.64$ & 0.939 \\
\hline ASA grading & & & & 0.020 \\
\hline 1 & $2(9.5)$ & $1(4.5)$ & $3(13.6)$ & \\
\hline$\|$ & $11(52.4)$ & 18 (81.8) & $19(86.4)$ & \\
\hline III & $8(38.1)^{*}$ & $3(13.6)$ & $0(0.0)$ & \\
\hline Epidural steroid injection (ESI) & $27.3^{*}$ & 4.5 & 0.0 & 0.007 \\
\hline No ESI & $16^{*}$ & 21 & 22 & 0.026 \\
\hline $1 \mathrm{ESI}$ & $4^{*}$ & 0 & 0 & \\
\hline $2 \mathrm{ESI}$ & 2 & 1 & 0 & \\
\hline Length of operation (min) & $165.23 \pm 52.45$ & $170.68 \pm 54.58$ & $160.27 \pm 43.06$ & 0.387 \\
\hline Length of hospital stay (day) & $6.10 \pm 7.85^{*}$ & $3.19 \pm 2.06$ & $2.71 \pm 0.75^{*}$ & 0.042 \\
\hline Fluoroscopy time (sec) & $50.91 \pm 24.07$ & $67.55 \pm 31.64$ & $62.91 \pm 28.19$ & 0.518 \\
\hline Time to ambulate (day) & $1.68 \pm 0.84^{*, * *}$ & $1.23 \pm 0.43^{*}$ & $1.14 \pm 0.35^{* *}$ & 0.006 \\
\hline Postoperative analgesia (morphine) (mg) & $10.16 \pm 10$ & $10.59 \pm 7.08$ & $8.62 \pm 6.92$ & 0.297 \\
\hline \multicolumn{5}{|l|}{ Type of bone graft (\%) } \\
\hline Local bone & 54.5 & 63.6 & 72.7 & 0.304 \\
\hline Infuse & 22.7 & 31.8 & 13.6 & \\
\hline DBM & 22.7 & 4.5 & 13.6 & \\
\hline Mean follow-up (mo) & $49.41 \pm 20.83$ & $49.77 \pm 19.67$ & $47.18 \pm 20.01$ & 0.899 \\
\hline
\end{tabular}

Values expressed as mean \pm standard deviation or number (\%).

$\mathrm{BMI}$, body mass index; ASA, American Society of Anesthesiologists; DBM, demineralized bone matrix.

${ }^{*, * *}$ Only post hoc comparisons between data across row are statistically significant.

group. Chronic smoking was observed in three patients (one in each group) ( $p>0.05)$. Minimum follow up period was two years postoperatively (range, $2-5.96$ years). The most common medical co-morbidity was hypertension, followed by hyperlipidemia and diabetes mellitus; the distribution of medical comorbidities amongst the three groups is presented in Table 2. Preoperative osteoporosis screening is not performed routinely at our institute. The majority of patients in the three groups fell into ASA category I/ II, with no significant difference in distribution amongst the three groups. There were eight patients in the "old-old" group and three patients in the "young-old" group who were classified in the ASA III category; which was statistically significant $(p=0.002)$.
Patients with advanced age showed a higher incidence of treatment with ESI preoperatively $(p<0.05)$. Four patients from the "old-old" age group received only one ESI while two patients from the "old-old" age group and one patient from the "young-old" received two ESIs. Symptom free interval after ESI was limited to a few months (range, 0-6 months) after the procedure.

There was no significant difference in perioperative outcomes between the groups except for the length of hospital stay $(p=0.042)$ and the time to ambulation $(p=0.006)$. The difference in these two parameters was observed between "old-old" and "middle-age" groups, and favored the "middle-age" group.

All patient groups showed significant improvement in 
Table 2. Distribution of medical co-morbidities

\begin{tabular}{lccc} 
Comorbidities & Old-old $(\mathrm{n}=22)$ & Young-old $(\mathrm{n}=22)$ & Middle age $(\mathrm{n}=22)$ \\
No Comorbidity & 2 & 2 & 1 \\
DM & 5 & 6 & 3 \\
HTN & 14 & 16 & 10 \\
HLD & 8 & 11 & 8 \\
\hline HD and TIA & 3 & 4 & 0 \\
Asthma & 0 & 1 & 1 \\
Cancer & 4 & 2 & 2 \\
Arthritis & 0 & 1 & 6 \\
\hline Dyspepsia & 4 & 3 & 1 \\
\hline Endocrine abnormalities & 2 & 0 & 1 \\
\hline Others & 2 & 1 & 2 \\
\hline
\end{tabular}

DM, diabetes mellitus; HTN, hypertension; HLD, hyperlipidemia; IHD, ischemic heart disease; TIA, transient ischemic attack.

Table 3. Clinical outcome scores

\begin{tabular}{|c|c|c|c|c|}
\hline Clinical outcome score & $\begin{array}{l}\text { Old-old } \\
(n=22)\end{array}$ & $\begin{array}{l}\text { Young-old } \\
(n=22)\end{array}$ & $\begin{array}{l}\text { Middle-age } \\
\quad(n=22)\end{array}$ & $\begin{array}{c}\text { Level of significance } \\
\text { between } 3 \text { groups } \\
p \text {-value }\end{array}$ \\
\hline \multicolumn{5}{|l|}{ ODI } \\
\hline Preoperative & $54.91 \pm 14.21 *$ & $43.81 \pm 14.93^{*}$ & $43.72 \pm 17.32$ & 0.028 \\
\hline 2-Year postoperative & $18.45 \pm 14.15$ & $20.34 \pm 17.42^{*}$ & $9.07 \pm 6.77^{*}$ & 0.017 \\
\hline \multicolumn{5}{|l|}{ VAS back pain } \\
\hline Preoperative & $5.32 \pm 3.06$ & $5.23 \pm 2.91$ & $6.64 \pm 2.61$ & 0.197 \\
\hline 2-Year postoperative & $1.05 \pm 2.32$ & $1.68 \pm 2.92$ & $0.50 \pm 1.47$ & 0.245 \\
\hline \multicolumn{5}{|l|}{ VAS leg pain } \\
\hline Preoperative & $5.18 \pm 3.67$ & $6.32 \pm 2.92$ & $6.09 \pm 2.89$ & 0.460 \\
\hline 2-Year postoperative & $0.86 \pm 2.36$ & $0.86 \pm 2.23$ & $0.32 \pm 1.29$ & 0.587 \\
\hline \multicolumn{5}{|l|}{ SF-36 physical function } \\
\hline Preoperative & $27.50 \pm 23.69 *$ & $37.95 \pm 27.63$ & $52.05 \pm 21.97^{*}$ & 0.006 \\
\hline 2-Year postoperative & $57.27 \pm 27.1^{*}$ & $63.41 \pm 21.12^{* *}$ & $79.55 \pm 11.64^{* * * *}$ & 0.002 \\
\hline \multicolumn{5}{|l|}{ SF-36 mental function } \\
\hline Preoperative & $67.64 \pm 19.67$ & $69.64 \pm 18.38$ & $74.91 \pm 17.51$ & 0.410 \\
\hline 2-Year postoperative & $82.00 \pm 18.13$ & $82.91 \pm 15.28$ & $85.64 \pm 13.95$ & 0.733 \\
\hline \multicolumn{5}{|l|}{ Neurogenic symptom score } \\
\hline Preoperative & $53.64 \pm 23.97$ & $47.73 \pm 24.59$ & $48.79 \pm 23.83$ & 0.689 \\
\hline 2-Year postoperative & $14.85 \pm 16.39$ & $11.97 \pm 18.01$ & $6.52 \pm 10.16$ & 0.191 \\
\hline
\end{tabular}

All clinical outcomes for all 3 groups demonstrated significant improvement $(p<0.005)$ between preoperative and 2 year postoperative scores. ODI, Oswestry disability index; VAS, visual analogue scale; SF-36, 36-item short form health survey.

${ }^{* * * *}$ Only post hoc comparisons between data with across row are statistically significant.

all clinical outcome scores postoperatively at all timepoints compared to preoperative data. Patients in the "middle age" group had better ODI scores $(p<0.05)$ and
SF-36 physical function score $(p<0.05)$ than patients in the "old-old" group; both preoperatively and at two years postoperatively. Mean clinical outcome scores are sum- 


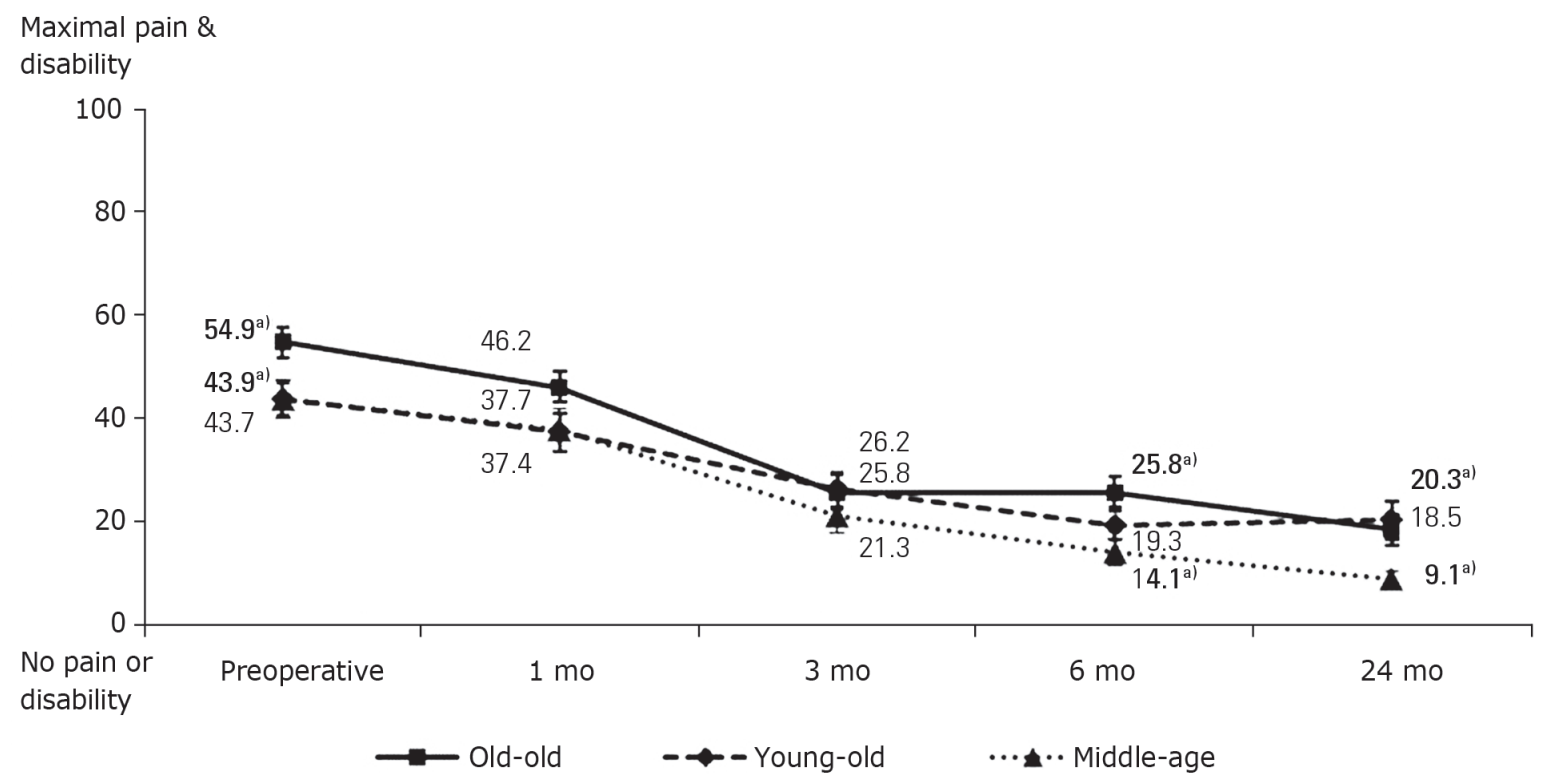

Fig. 1. Trends in Oswestry disability index up to the two year follow-up. ${ }^{\text {al }} \mathrm{A}$ significant difference $(p<0.05)$ between the means of the 'marked' age groups at the respective time-point.

Worst possible pain

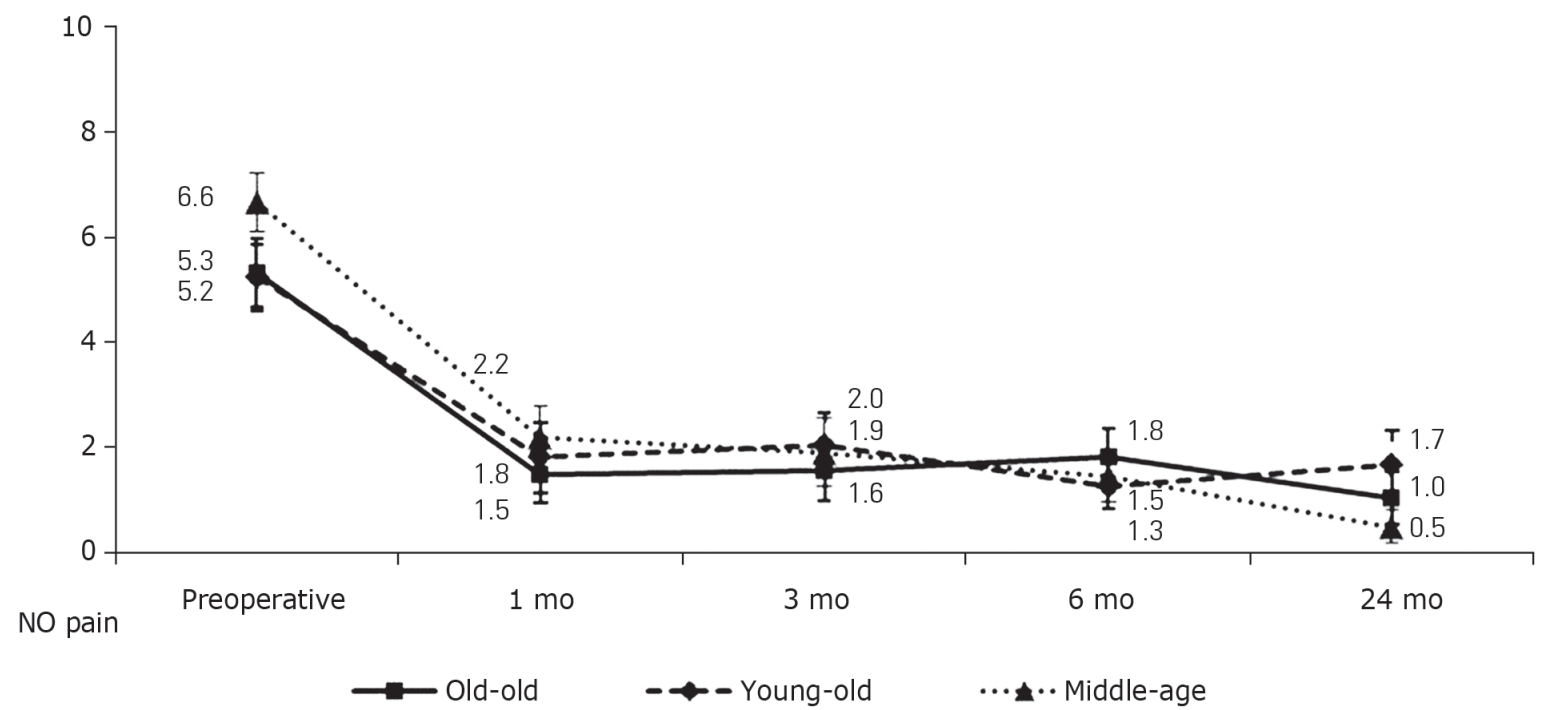

Fig. 2. Trends in visual analogue scale back pain up to the two year follow-up.

marized in Table 3. Trends of improvement in clinical outcome measures can be seen graphically in Figs. 1-6. More than $50 \%$ of patients in all three groups achieved MCID levels in all clinical outcomes by as early as six months postoperatively. The proportion of patients achieving MCID levels in our study ranged from $63 \%-95 \%$ for the respective scores at two years postoperatively. There was no statistically significant difference $(p>0.05)$ in MCID levels of any clinical outcome scores between the three groups at every follow up visit. A graphical representation of the proportion (\%) of patients achieving MCID level is shown in Figs. 7 and 8.

Radiological outcomes are summarized in Table 4. More than $80 \%$ of patients in each age group demonstrated grade I radiological fusion at 2 year follow up without any significant difference between the groups $(p=0.422)$. 


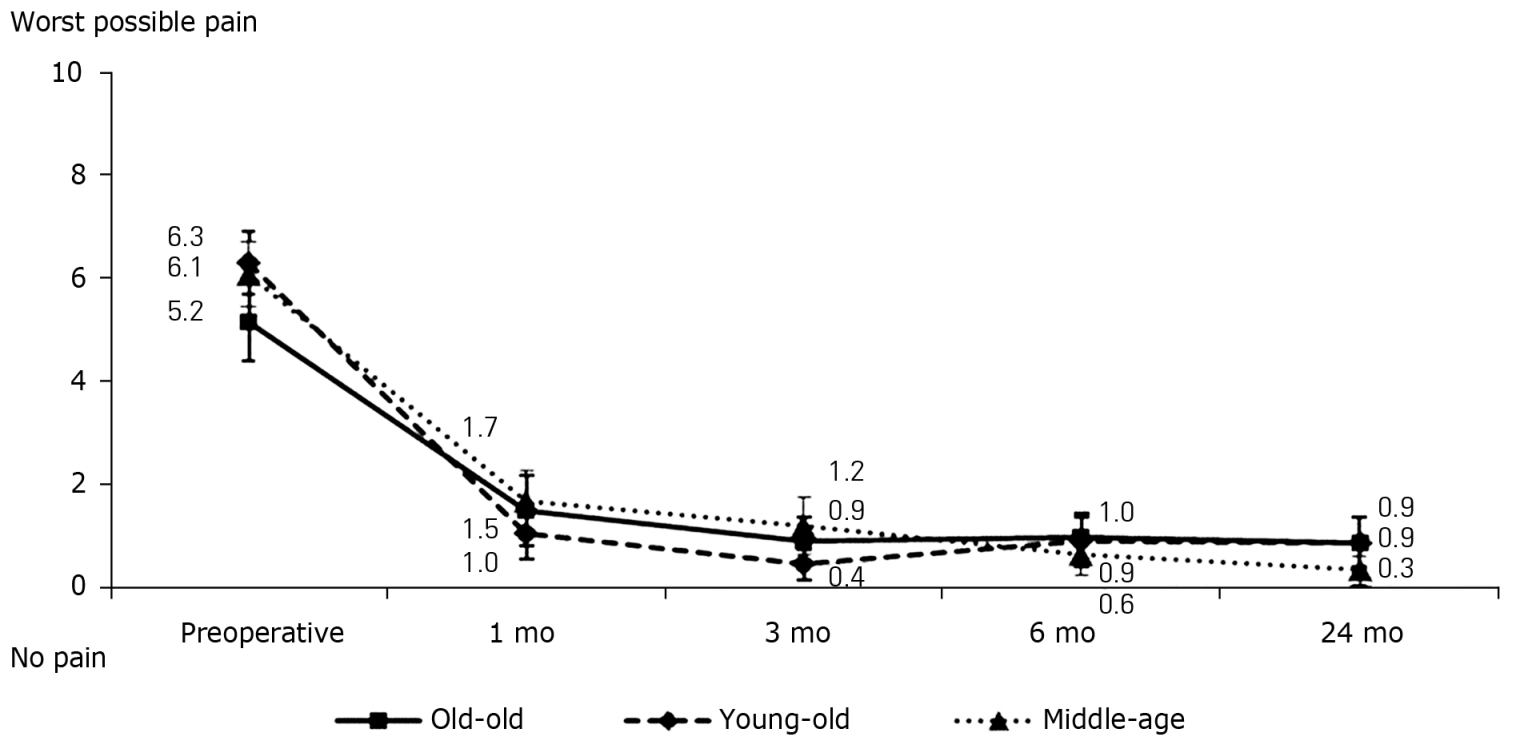

Fig. 3. Trends in visual analogue scale leg pain up to the 2-year follow-up.

\section{Excellent health}

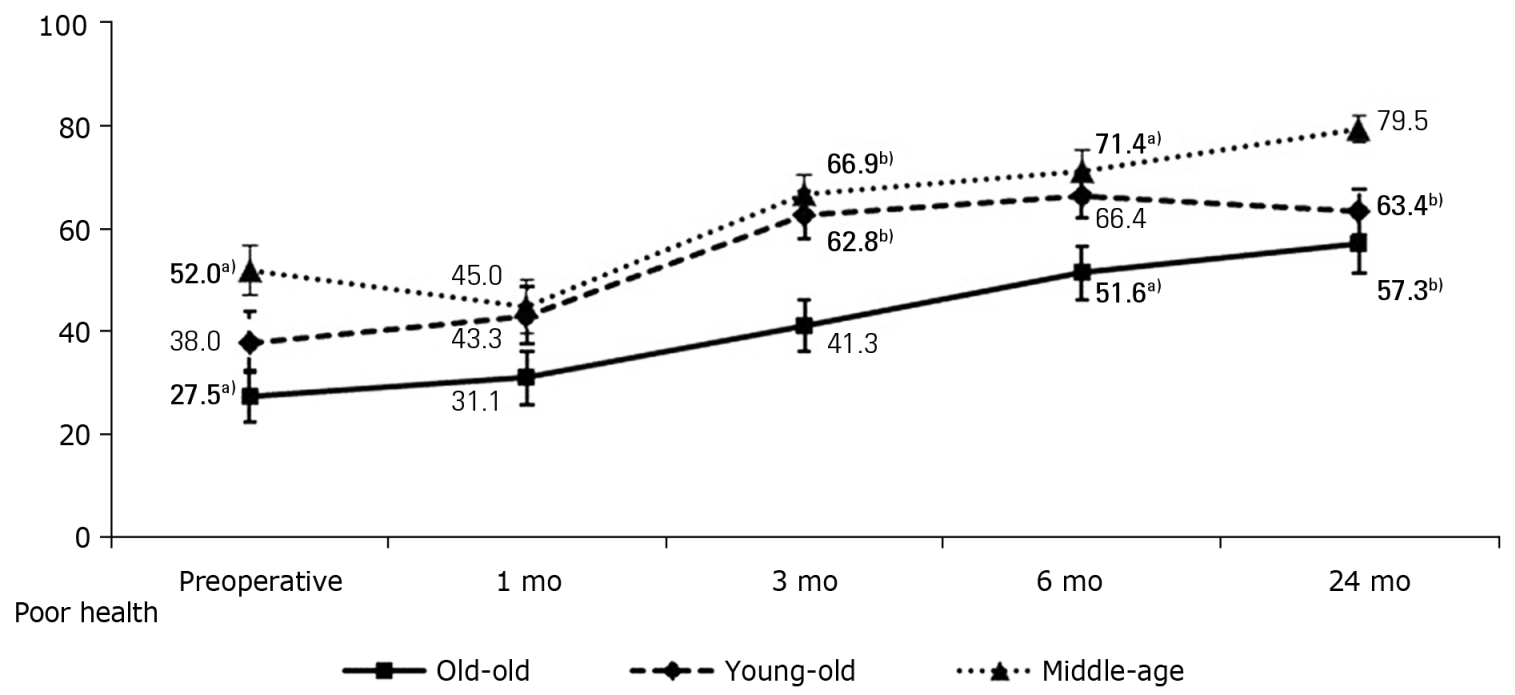

Fig. 4. Trends in 36-item short form health survey (SF-36) physical function up to the 2-year follow-up. a) A significant difference $(p<0.05)$ between the means of the 'marked' age groups at the respective time-point; ${ }^{b)} A$ significant difference $(p<0.05)$ between the means of the 'marked' and 'un-marked' age group at the respective time-point.

Screw malposition/loosening, cage migration/subsidence were purely radiological findings without any clinical significance ( $p>0.05)$; except in one patient from the "old-old" age group who required revision surgery within the same hospital admission due to postoperative radiculopathy secondary to a medially-placed pedicle screw. Radiological ASD was more common than clinical ASD, which was seen in two patients from the "young-old" group with no significant difference ( $p=0.255)$ between the three groups. Both of these patients were investigated with MRI and only one patient underwent surgery at the adjacent level four years after the index surgery; the other patient was treated with ESI. Cohen's kappa test revealed moderate to almost perfect agreement between two investigators 


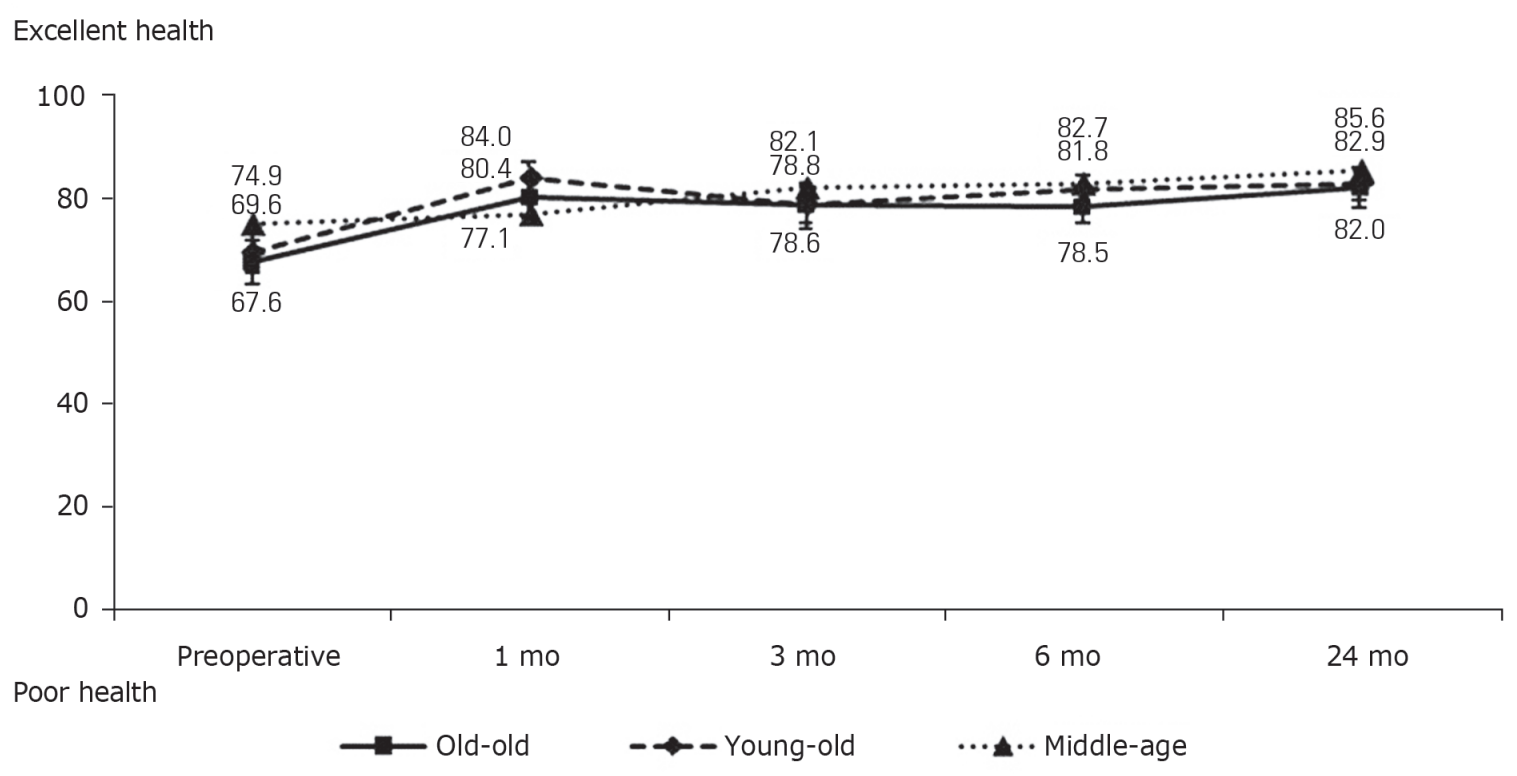

Fig. 5. Trends in 36-item short form health survey (SF-36) mental function up to the 2-year follow-up.

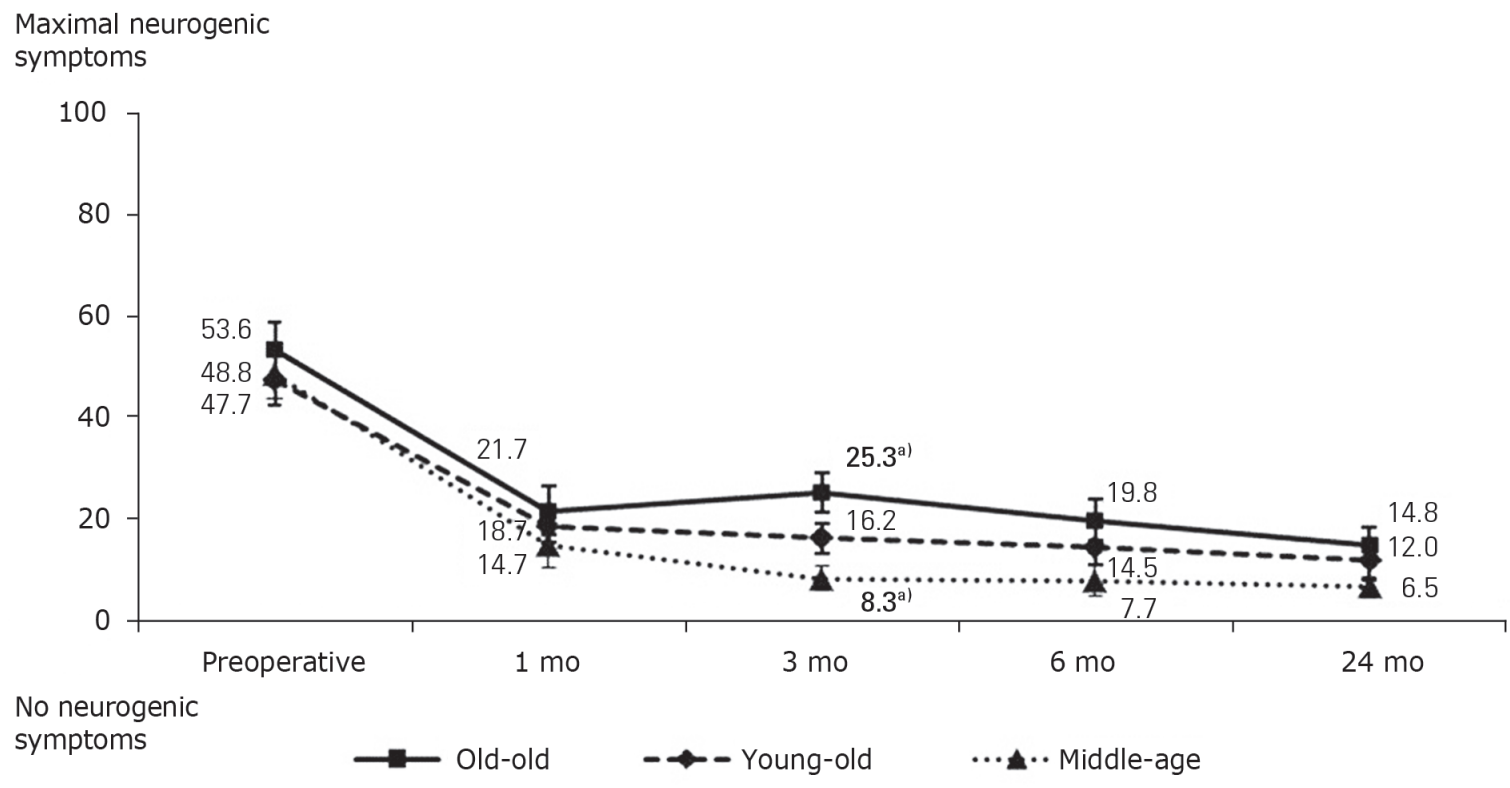

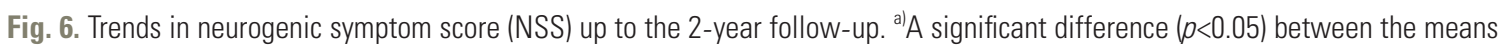
of the 'marked' age groups at the respective time-point.

$(p<0.005)$ in terms of radiological analysis.

Early major complications were seen in four cases from the "old-old" age group which included postoperative radiculopathy due to a medially-misdirected screw which required revision $(n=1)$, atrial fibrillation $(n=2)$ and acute delirium and renal dysfunction $(n=1)$. One symptomatic ASD patient from the "young-old" age group experienced a late major complication, and underwent surgery four years after index surgery. The most common minor complications were UTI (three patients from the "old-old" and one patient from the "young-old" age group) and superficial vein thrombosis (two each from the "old-old" and "young-old" age group). There was one incidence of durotomy in a patient from the "young-old" age group which was sealed intraoperatively using fibrin sealant without any postoperative consequences. The incidence 


\section{Oswestry disability index}

(\% of patients met MCID)

(\%)

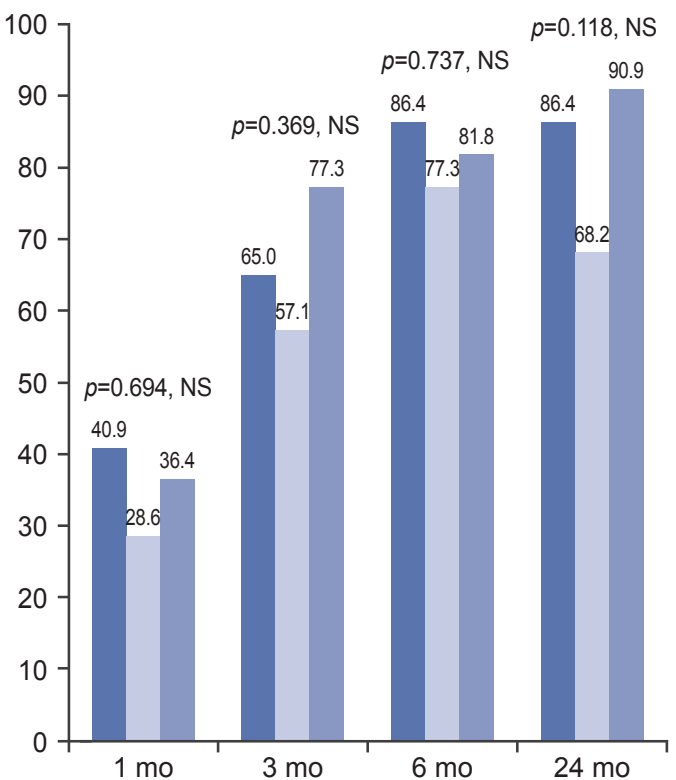

SF-36 PCS

(\% of patients met MCID)

(\%)

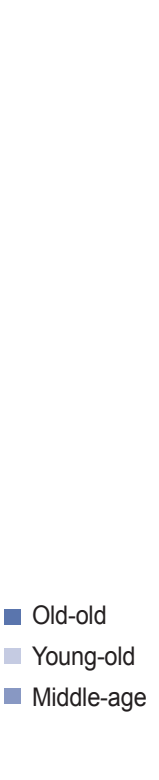

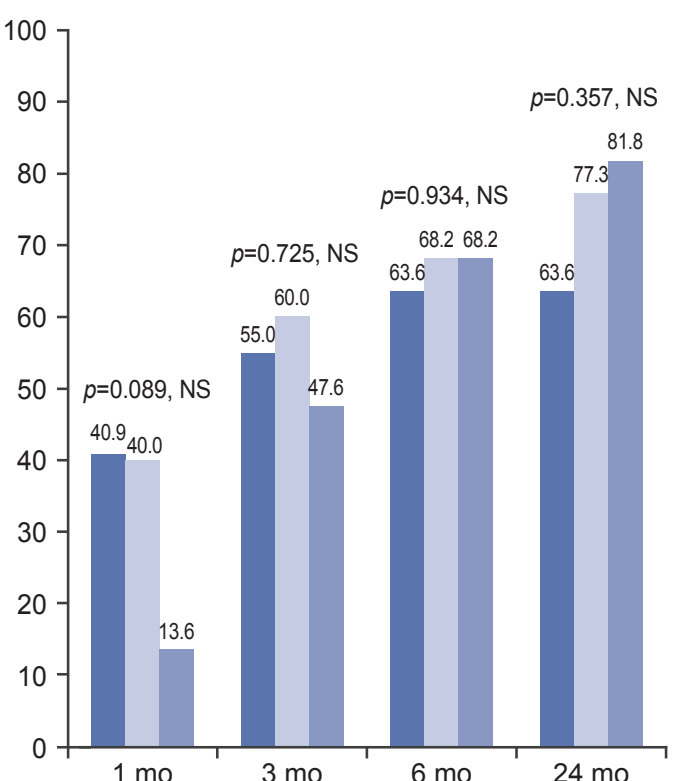

Fig. 7. Graphical representation of the proportion (\%) of patients attaining minimal clinically important difference (MCID) levels for Oswestry disability index and 36-item short form health survey (SF-36) physical function at each follow-up visit. The ' $p$-value' at the top of each of the three columns indicates the level of significance between the three age groups at every follow up visit. NS, not significant.

VAS back pain

(\% of patients met MCID)

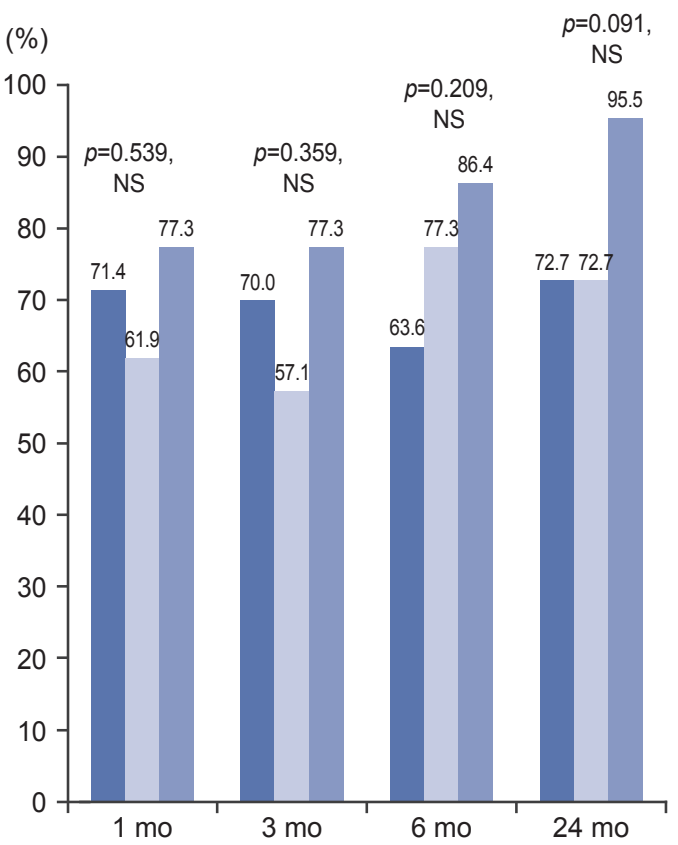

VAS leg pain

(\% of patients met MCID)

(\%)

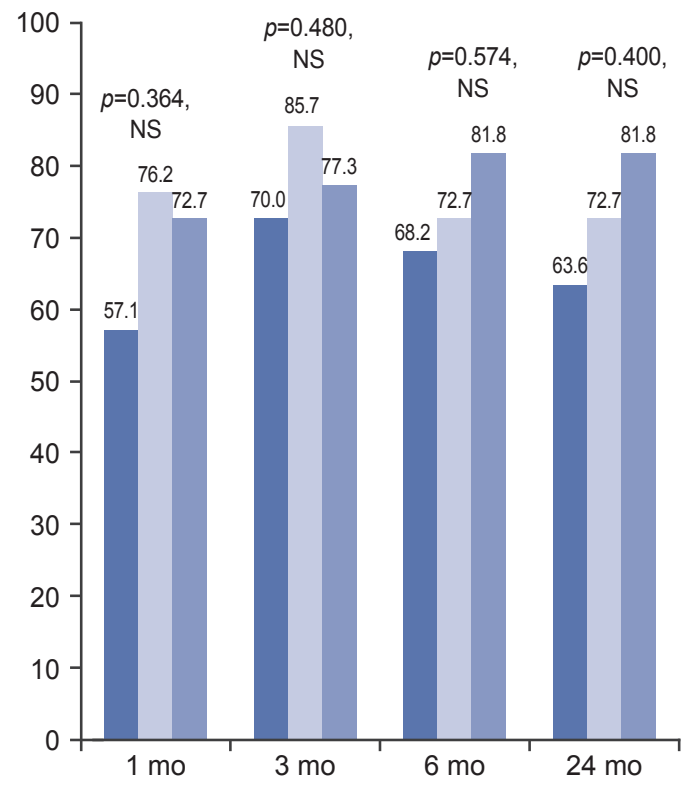

Fig. 8. Graphical representation of the proportion (\%) of patients attaining minimal clinically important difference (MCID) levels for visual analogue scale (VAS) back pain and leg pain at each follow-up visit. The ' $p$-value' at the top of each of the three columns indicates the level of significance between the three age groups at every follow-up visit. NS, not significant. 
Table 4. Radiologic outcomes at 2 years postoperatively

\begin{tabular}{|c|c|c|c|c|}
\hline Radiologic criteria & $\begin{array}{l}\text { Old-old } \\
(n=22)\end{array}$ & $\begin{array}{l}\text { Young-old } \\
(n=22)\end{array}$ & $\begin{array}{l}\text { Middle-age } \\
\quad(n=22)\end{array}$ & $\begin{array}{c}\text { Level of } \\
\text { significance } \\
p \text {-value }\end{array}$ \\
\hline Fusion (\%) (Bridwell et al. [18]) & & & & 0.422 \\
\hline 1 & 80 & 83.3 & 90 & \\
\hline$\|$ & 10 & 13.3 & 10 & \\
\hline III & 10 & 3.3 & 0 & \\
\hline Screw loosening (\%) & & & & 0.680 \\
\hline Absent & 81.8 & 86.4 & 90.9 & \\
\hline Present & 18.2 & 13.6 & 9.1 & \\
\hline Screw malposition (\%) & & & & 0.766 \\
\hline Absent & 90.9 & 95.5 & 95.5 & \\
\hline Present & 9.1 & 4.5 & 4.5 & \\
\hline Cage migration (\%) & & & & 0.766 \\
\hline Absent & 90.9 & 95.5 & 95.5 & \\
\hline Present & 9.1 & 4.5 & 4.5 & \\
\hline Cage subsidence (\%) & & & & 0.345 \\
\hline Absent & 90.9 & 90.9 & 100 & \\
\hline Present & 9.1 & 9.1 & 0 & \\
\hline Adjacent segment degeneration $(\%)[19,20]$ & & & & 0.255 \\
\hline No ASD & 50 & 31.8 & 50 & \\
\hline Radiologic ASD & 50 & 59.1 & 50 & \\
\hline Clinical ASD & 0 & 9.1 & 0 & \\
\hline
\end{tabular}

ASD, adjacent segment degeneration.

Table 5. Incidence of complications (\%) in each group at 2-year follow up

\begin{tabular}{|c|c|c|c|c|c|c|}
\hline \multirow{2}{*}{ Variable } & \multicolumn{3}{|c|}{ Major complications (\%) } & \multicolumn{3}{|c|}{ Minor complications (\%) } \\
\hline & No complication & Early & Late & No complication & Early & Late \\
\hline Old-old & 18 (81.8) & $4(18.2)$ & 0 & $14(63.6)$ & $7(31.9)$ & $1(4.5)$ \\
\hline Young-old & $21(95.5)$ & 0 & $1(4.5)$ & $12(54.6)$ & $9(40.9)$ & $1(4.5)$ \\
\hline Middle-age & $22(100)$ & 0 & 0 & $19(86.4)$ & $3(13.6)$ & 0 \\
\hline Level of significance $p$-value & \multicolumn{3}{|c|}{0.205 , Not significant } & \multicolumn{3}{|c|}{0.120, Not significant } \\
\hline
\end{tabular}

of complications are summarized in Table 5. There was no statistical difference $(p>0.05)$ between the three groups with respect to the incidence of major/minor complications.

\section{Discussion}

The incidence of morbidity and mortality associated with spine surgery in the elderly is well documented [3-6].
While older literature is more focused upon the incidence of perioperative complications, more recent literature highlights the clinical outcomes along with complications. Fujita et al. [3] reported a high incidence (62.5\%) of major complications after spinal fusion for deformity in patients more than 60 years and an incidence of $33 \%$ in terms of major complications in patients undergoing fusion for lumbar stenosis. Oldridge et al. [5] concluded that 
patients in excess of 80 years of age face the highest risk of increased morbidity and mortality $(>10 \%)$ in cases of spinal fusion. Okuda et al. [21] evaluated clinical and radiological outcomes of instrumented PLIF in the elderly; clinical improvement, as measured by the Japanese Orthopaedic Association score, was significant in both elderly and younger populations following surgery without any statistically significant difference between the two groups. Glassman et al. [8] demonstrated similar outcomes following PLF in younger ( $<65$ years) and older ( $>65$ years) patients when assessed two years post surgery despite a higher rate of complications (56\%) in older patients during the perioperative period; these authors concluded that perioperative complications did not appear to adversely affect clinical outcomes two years post surgery.

While the majority of these previous studies involved open surgical techniques, there is scant evidence available with regard to the use of minimally invasive techniques in elderly patients with spinal pathologies [14,22,23]. Lee et al. [23] concluded that single level mini-open TLIF yielded good clinical and radiological outcomes with fewer perioperative complications in patients $>65$ years of age when assessed three years post surgery. Karikari et al. [14] further reported an overall low rate of major complications in patients more than 70 years treated by either extreme lateral interbody fusion or MIS-TLIF.

To the best of our knowledge, very few studies have reported the outcomes of MIS-TLIF in the elderly population, especially in comparison to other age groups. MISTLIF has several inherent advantages, including reduced blood loss and fewer injuries to the paraspinal musculature, leading to a reduction in postoperative pain and the reduced use of postoperative analgesia. These factors allow patients to mobilize earlier leading to shorter hospital stays [9-12]. These factors are especially helpful to elderly patients as most perioperative complications are the result of prolonged immobilization secondary to pain and blood loss.

ESI is an important aspect of the conservative care of spinal pathologies and is very commonly used in elderly patients who are either not medically fit to undergo surgery or who are denied surgical options due to advanced age. Recent literature has concluded that previous ESIs are associated with less improvement in patients undergoing fusion surgery for lumbar stenosis at a later date [24]. In our present study, we observed that the majority of ESIs were received by patients in the older age groups. There was no difference in clinical and radiological outcomes in those who did/did not have ESI as part of their pre-surgical treatment. We also found that patients from the "oldold" age group took more time for mobilization and had longer hospital stays than "middle-age" patients. This is likely to be due to elderly patients in our population being fearful of early mobilization after major spine surgery and hesitancy on the part of their caregivers in taking them home early.

All of our patients showed significant improvement in clinical outcome scores; these findings are comparable with those of previous studies $[13,14]$. In our study, grade I fusion was achieved in $80 \%-90 \%$ patients at the end of the 2-year follow-up; this was in accordance with existing literature $[9,11]$. There was no significant difference between the three study groups with regard to bone graft material and major complications were in the range of $0-18 \%$. Minor complications were more common, occurring in $0 \%-36 \%$ of cases $[9,11-13]$. More complications were observed in the "old-old" and "young-old" patients, although this observation was not statistically significant and did not influence clinical outcomes. Radiological ASD was much more common than clinical ASD, at incidences of $50 \%-59 \%$ and $0 \%-9.1 \%$, respectively. These findings were similar to the incidence of radiological ASD $(5.2 \%-100 \%)$ and clinical ASD (5.2\%-30.3\%) reported in the previous literature [13].

There are some limitations to our study, which should be considered when interpreting our results. First, this study involved a matched pair analysis and not a randomized prospective study; this was a retrospective study with a relatively small number of patients. Second, perioperative outcomes such as the length of hospitalization and the time for ambulation could have been affected by external factors such as variations in physiotherapists, nurses, and patient expectations. Third, we experienced more patients with ASA 3 in the old-old group. Despite this, these patients were able to achieve comparable clinical outcomes and complication rates with patients in the other younger age-groups. Computed tomography scans were not used as part of our standard evaluation of fusion status due to cost concerns. Measures undertaken to reduce potential bias included the patient population being assigned to a single surgeon from a single institute, obtaining independent assessors to assess clinical outcome scores, and using Cohen's kappa test to rule out inter-observer bias. All functional outcome scores were prospectively recorded 
and form part of our registry data for surgical spine procedures in our institution.

\section{Conclusions}

MIS-TLIF has comparable results in "old-old" and "young-old" patients when compared with "middle-age" patients. Therefore, MIS-TLIF can be considered a safe and effective procedure for elderly patients with predominant neurogenic symptoms and instability in which adequate conservative treatment has failed.

\section{Conflict of Interest}

The senior author (W.M.Y.) is a consultant for Medtronic and Depuy Synthes; but no funding was received for purposes of this study. All other authors have no conflicts of interest or sources of funding to disclose.

The legal regulatory status of the device(s)/drug(s) that is/are the subject of this manuscript is not applicable in my country.

\section{ORCID}

Joshi Nikhil N : 0000-0002-9736-0939

Lim Wei-An Joel: 0000-0002-8765-2858

William Yeo: 0000-0003-4143-6989

Wai Mun Yue: 0000-0002-7330-8159

\section{References}

1. Deyo RA, Ciol MA, Cherkin DC, Loeser JD, Bigos SJ. Lumbar spinal fusion: a cohort study of complications, reoperations, and resource use in the Medicare population. Spine (Phila Pa 1976) 1993;18:1463-70.

2. Deyo RA, Cherkin DC, Loeser JD, Bigos SJ, Ciol MA. Morbidity and mortality in association with operations on the lumbar spine: the influence of age, diagnosis, and procedure. J Bone Joint Surg Am 1992;74: 536-43.

3. Fujita T, Kostuik JP, Huckell CB, Sieber AN. Complications of spinal fusion in adult patients more than 60 years of age. Orthop Clin North Am 1998;29:66978.

4. Raffo CS, Lauerman WC. Predicting morbidity and mortality of lumbar spine arthrodesis in patients in their ninth decade. Spine (Phila Pa 1976) 2006;31:99-
103.

5. Oldridge NB, Yuan Z, Stoll JE, Rimm AR. Lumbar spine surgery and mortality among Medicare beneficiaries, 1986. Am J Public Health 1994;84:1292-8.

6. Carreon LY, Puno RM, Dimar JR 2nd, Glassman SD, Johnson JR. Perioperative complications of posterior lumbar decompression and arthrodesis in older adults. J Bone Joint Surg Am 2003;85:2089-92.

7. Vitaz TW, Raque GH, Shields CB, Glassman SD. Surgical treatment of lumbar spinal stenosis in patients older than 75 years of age. J Neurosurg 1999;91(2 Suppl):181-5.

8. Glassman SD, Polly DW, Bono CM, Burkus K, Dimar JR. Outcome of lumbar arthrodesis in patients sixtyfive years of age or older. J Bone Joint Surg Am 2009; 91:783-90.

9. Peng CW, Yue WM, Poh SY, Yeo W, Tan SB. Clinical and radiological outcomes of minimally invasive versus open transforaminal lumbar interbody fusion. Spine (Phila Pa 1976) 2009;34:1385-9.

10. Isaacs RE, Podichetty VK, Santiago P, et al. Minimally invasive microendoscopy-assisted transforaminal lumbar interbody fusion with instrumentation. J Neurosurg Spine 2005;3:98-105.

11. Lee KH, Yue WM, Yeo W, Soeharno H, Tan SB. Clinical and radiological outcomes of open versus minimally invasive transforaminal lumbar interbody fusion. Eur Spine J 2012;21:2265-70.

12. Seng C, Siddiqui MA, Wong KP, et al. Five-year outcomes of minimally invasive versus open transforaminal lumbar interbody fusion: a matched-pair comparison study. Spine (Phila Pa 1976) 2013;38:204955.

13. Kim JS, Jung B, Lee SH. Instrumented minimally invasive spinal-transforaminal lumbar interbody fusion (MIS-TLIF): minimum 5-years follow-up with clinical and radiologic outcomes. J Spinal Disord Tech. 2012 Sep 28 [Epub]. https://doi.org/10.1097/ BSD.0b013e31827415cd.

14. Karikari IO, Grossi PM, Nimjee SM, et al. Minimally invasive lumbar interbody fusion in patients older than 70 years of age: analysis of peri- and postoperative complications. Neurosurgery 2011;68:897-902.

15. Copay AG, Glassman SD, Subach BR, Berven S, Schuler TC, Carreon LY. Minimum clinically important difference in lumbar spine surgery patients: a choice of methods using the Oswestry Disability 
Index, Medical Outcomes Study questionnaire Short Form 36, and pain scales. Spine J 2008;8:968-74.

16. Parker SL, Adogwa O, Paul AR, et al. Utility of minimum clinically important difference in assessing pain, disability, and health state after transforaminal lumbar interbody fusion for degenerative lumbar spondylolisthesis. J Neurosurg Spine 2011;14:598604.

17. Lebude B, Yadla S, Albert T, et al. Defining "complications" in spine surgery: neurosurgery and orthopedic spine surgeons' survey. J Spinal Disord Tech 2010; 23:493-500.

18. Bridwell KH, Lenke LG, McEnery KW, Baldus C, Blanke K. Anterior fresh frozen structural allografts in the thoracic and lumbar spine: do they work if combined with posterior fusion and instrumentation in adult patients with kyphosis or anterior column defects? Spine (Phila Pa 1976) 1995;20:1410-8.

19. Bae JS, Lee SH, Kim JS, Jung B, Choi G. Adjacent segment degeneration after lumbar interbody fusion with percutaneous pedicle screw fixation for adult low-grade isthmic spondylolisthesis: minimum 3 years of follow-up. Neurosurgery 2010;67:1600-7.

20. Cheh G, Bridwell KH, Lenke LG, et al. Adjacent segment disease following lumbar/thoracolumbar fusion with pedicle screw instrumentation: a minimum 5-year follow-up. Spine (Phila Pa 1976) 2007;32:22537.

21. Okuda S, Oda T, Miyauchi A, Haku T, Yamamoto T, Iwasaki M. Surgical outcomes of posterior lumbar interbody fusion in elderly patients. J Bone Joint Surg Am 2006;88:2714-20.

22. Lee P, Fessler RG. Perioperative and postoperative complications of single-level minimally invasive transforaminal lumbar interbody fusion in elderly adults. J Clin Neurosci 2012;19:111-4.

23. Lee DY, Jung TG, Lee SH. Single-level instrumented mini-open transforaminal lumbar interbody fusion in elderly patients. J Neurosurg Spine 2008;9:137-44.

24. Radcliff K, Kepler C, Hilibrand A, et al. Epidural steroid injections are associated with less improvement in patients with lumbar spinal stenosis: a subgroup analysis of the Spine Patient Outcomes Research Trial. Spine (Phila Pa 1976) 2013;38:279-91. 